\title{
铜离子抑制腺苷同型半胱氨酸水解酶的活性
}

\author{
陈劼苓 刘青宇 杨晓达* 王 尊 \\ (北京大学药学院化学生物学系, 天然药物及仿生药物国家重点实验室, 北京 100083.* 联系人, E-mail: xiaodayang@ hotmail.com)
}

\begin{abstract}
摘要 利用 E. coli JM109 菌株高效表达和纯化了重组人的 S-腺苷同型半胱氨酸(AdoHcy)水解酶. 初步 研究了铜离子对 AdoHcy 水解酶活性的影响. 结果表明, $\mathrm{Cu}^{2+}$ 可以抑制酶的活性, 其抑制能力随着 $\mathrm{Cu}^{2+}$ 浓度增加而增大; $\mathrm{Cu}^{2+}$ 对 AdoHcy 水解酶活性抑制是一个时间依赖的过程, 其表观速率常数为(1.08土 $0.15) \mathrm{min}^{-1}$. 自然底物腺苷(Ado)的存在可以明显减弱 $\mathrm{Cu}^{2+}$ 抑制酶活性的能力. 研究结果表明, $\mathrm{Cu}^{2+}$ 可能 与酶的自然底物以某种类似方式竞争结合，从而抑制酶的活性.
\end{abstract}

\section{关键词 $\mathrm{Cu}^{2+} \mathrm{S}$-腺苷同型半胱氨酸水解酶 生物甲基化}

S-腺苷同型半胱氨酸 (S-Adenosylhomocysteine, AdoHcy)水解酶催化 AdoHcy 水解生成腺苷(Adenosine, Ado) 和同型半胱氨 酸 (Homocysteine, Hcy $)^{[1,2]}$. AdoHcy 是腺苷基蛋氨酸(Adenosylmethionine, AdoMet) 在生物甲基化反应后的产物, 它强烈地抑制各种 AdoMet 依赖的生物甲基化过程 ${ }^{[1,2]}$; 另一方面, 血清 $\mathrm{Hcy}$ 水平过高已经被证明是导致心血管疾病的独立 危险因子 ${ }^{[3]}$. 因此, AdoHcy 水解酶是控制细胞生物甲 基化和 Hcy 代谢等生理过程的一个重要酶 ${ }^{[1,2]}$. 长久 以来, AdoHcy 水解酶成为药物相关研究中非常重要 的靶分子, 用于抗癌 ${ }^{[4]}$ 、抗病毒、抗寄生虫、抗关节 炎和治疗心血管疾病等的药物设计 ${ }^{[1,2]}$. 最近, AdoHcy 水解酶的晶体结构也被成功解析 ${ }^{[5,6]}$. 它的结 构和催化机理已经得到了十分深人的阐述 ${ }^{[7-11]}$.

虽然 AdoHcy 水解酶的结构和催化作用并不需 要金属离子 ${ }^{[111]}$, 但是有证据显示金属离子可能参与 细胞 AdoHcy 水解酶活性的调节 ${ }^{[12 \sim 18]}$. Suarez 等人 ${ }^{[12]}$ 发现肾上腺素可通过钙调节途径影响 AdoHcy 水解 酶的活性, 提示细胞可能通过金属离子如钙离子作 用影响该水解酶. 不过 Kloor 等人 ${ }^{[13]}$ 的研究表明, 在 生理条件下䥻离子还不足以产生显著影响. Ettinger 等人 ${ }^{[14]}$ 发现 AdoHcy 水解酶能够紧密和可逆地结合 铜离子 ${ }^{[14 ~ 16]}$, 解离常数达 $K_{\mathrm{d}}=10^{-16} \mathrm{~mol} \cdot \mathrm{L}^{-1}$, 并且组 织中铜的水平与 AdoHcy 水解酶表达水平是一致 的 ${ }^{[14 ~ 16]}$. 因此, AdoHcy 水解酶有可能是一种铜离子 的新载体, 影响铜的代谢和分配. 但是, 并不清楚铜 离子如何特异性地结合 AdoHcy 水解酶以及对酶活 性有何影响. 近年来, 有研究发现铜离子可增加细胞 $\mathrm{Hcy}$ 的释放 ${ }^{[17]}$ 以及高血清 Hcy 水平伴随着血清铜浓 度的升高 ${ }^{[18]}$. 总之, 这些迹像表明铜离子有可能是
调节体内 AdoHcy 水解酶活性水平的一个因素. 而铜 离子可能调控 AdoHcy 水解酶的活性显然也关联到 铜络合物抗病毒和抗炎等药理作用的机理.

本文报道铜离子对 AdoHcy 水解酶作用及其机 理的初步研究结果. 我们发现 $\mathrm{Cu}^{2+}$ 可以抑制 AdoHcy 水解酶的活性，活性抑制是一个浓度和时间依赖的 过程; 此外, Ado 可以显著减弱 $\mathrm{Cu}^{2+}$ 抑制酶活性的能 力, 金属离子与酶的自然底物相竞争的现象是第一 次被观察到.

\section{1 材料与方法}

(i) 试剂. 转染编码腺苷同型半朕氨酸(AdoHcy) 水解酶的质粒(pPROK-1)的大肠杆菌 JM109 菌株由 美国 University of Kansas 惠赠. 腺苷购自上海丽珠东 风生物公司. 同型半胱氨酸和腺芺同型半胱氨酸购 自 Sigma 公司. 其他试剂均为国产分析纯.

(ii) AdoHcy 水解酶的提取与纯化. AdoHcy 水 解酶由转染了编码腺苷同型半胱氨酸水解酶的质粒 pPROK-1 的大肠杆菌 JM109 菌株按文献[19]方法表 达. 用离心 $\left(4^{\circ} \mathrm{C}, 6000 \mathrm{r} \cdot \mathrm{min}^{-1}, 15 \mathrm{~min}\right)$ 收集 JM109 细 胞并用生理盐水洗涤. 每升培养液用 $50 \mathrm{~mL} 20$ $\mathrm{mmol} \cdot \mathrm{L}^{-1}$ Tris- $\mathrm{HCl}(\mathrm{pH}$ 8) 溶液悬浮细菌细胞, 在冰水 浴中用超声粉碎细胞 $(400 \mathrm{~W}, 3 \mathrm{~s} \times 140$, 间隔 $3 \mathrm{~s})$. 然 后离心 $\left(15000 \mathrm{r} \cdot \mathrm{min}^{-1} 60 \mathrm{~min}\right)$ 得到无细胞提取液 (CFE). AdoHcy 水解酶从 CFE 中经 DE-52 离子交换 $\left(1.5 \mathrm{~cm} \times 15 \mathrm{~cm}\right.$, 用 $10 \mathrm{mmol} \cdot \mathrm{L}^{-1} \mathrm{PBE}, \mathrm{pH} 7.2$ 预平衡 和洗涤, $20 \mathrm{mmol} \cdot \mathrm{L}^{-1} \mathrm{pH} 5.0$ 的苹果酸溶液洗脱, 流 速 $\left.3.5 \mathrm{~mL} \cdot \mathrm{min}^{-1}\right)$ 和 Sepharose 4B 凝胶过滤 $(2.5 \mathrm{~cm} \times$ $50 \mathrm{~cm}$, 用 $50 \mathrm{mmol} \cdot \mathrm{L}^{-1} \mathrm{PBE}, \mathrm{pH} 7.2$ 洗脱, 流速 1.0 $\mathrm{mL}^{13} \mathrm{~min}^{51}$ )两次柱色谱分离得到纯化后的酶制品. 
(iii) AdoHcy 水解酶浓度和纯度测定. AdoHcy 水解酶浓度按文献[20]方法用 $280 \mathrm{~nm}$ 波长吸收值大 小来测定. 蛋白纯度用 SDS-PAGE 电泳分析.

(iv) AdoHcy 水解酶活性的测定. 这里测定合成 方向 $(A d o+\mathrm{Hcy} \rightarrow$ AdoHcy, 一定时间内从 Ado 和 $\mathrm{Hcy}$ 合成 AdoHcy 的量)的酶活性: 在 $250 \mu \mathrm{L}$ Tris- $\mathrm{HCl}$ $\left(20 \mathrm{mmol} \cdot \mathrm{L}^{-1}, \mathrm{pH}\right.$ 7.4)溶液中, 加人 $10 \mu \mathrm{g}$ 的 AdoHcy 水解酶. 然后加人 $50 \mu \mathrm{L}$ Ado $\left(10 \mathrm{mmol} \cdot \mathrm{L}^{-1}\right), 200 \mu \mathrm{L}$ Hcy $\left(10 \mathrm{mmol} \cdot \mathrm{L}^{-1}\right)$, 于 $37^{\circ} \mathrm{C}$ 温育 $20 \mathrm{~min}$, 加人硫酸 $20 \mu \mathrm{L}$ 停止反应, 放人冰水中冷却, $10000 \mathrm{r} \cdot \mathrm{min}^{-1}$ 离 心 $5 \mathrm{~min}$. 进样量 $20 \mu \mathrm{L}$, 进行 HPLC 分析. 这里使用 了较文献[10]简化了的色谱条件： $4.6 \mathrm{~mm} \times 250 \mathrm{~mm}$ Diamonsil ${ }^{\mathrm{TM}} \mathrm{C} 18$ 柱, $\mathrm{A}$ 相为 $0.1 \%$ 磷酸溶液, $\mathrm{B}$ 相为乙 腈(色谱纯), A : B = 98:2, 流速 $1.00 \mathrm{~mL} \cdot \mathrm{min}^{-1}$.

(V) 铜离子对 AdoHcy 水解酶的活性的影响.

不同浓度铜离子的影响. 在 $250 \mu \mathrm{L}$ Tris- $\mathrm{HCl}$ (20 $\mathrm{mmol} \cdot \mathrm{L}^{-1}, \mathrm{pH}$ 7.4)溶液中, 用逐级稀释法制备不同终 浓度的铜离子溶液系列, 在其中加人 $10 \mu \mathrm{g}$ 酶, 于 $37^{\circ} \mathrm{C}$ 温育 $30 \mathrm{~min}$. 然后如上述(iv)方法用 HPLC 测定 酶合成方向的活性. (2) 铜离子与酶不同作用时间影 响. 铜离子溶液 $\left(1 \mu \mathrm{mol} \cdot \mathrm{L}^{-1}\right)$ 中加人 $10 \mu \mathrm{g}$ AdoHcy 水解酶, 分别于 $37^{\circ} \mathrm{C}$ 温育 $0,1,5,10,15$ 和 $20 \mathrm{~min}$. 然 后如上述方法测定酶的活性. (3) 腺苷存在下铜离子 的作用变化. 在 $240 \mu \mathrm{L} 0,10,100 \mathrm{nmol} \cdot \mathrm{L}^{-1}$ 及 1,10 , $100 \mu \mathrm{mol} \cdot \mathrm{L}^{-1}$ 的铜离子溶液中加人 $10 \mu \mathrm{g}$ 酶和 $\mathrm{Ado}($ 终 浓度 $1 \mathrm{mmol} \cdot \mathrm{L}^{-1}$ ), 于 $37^{\circ} \mathrm{C}$ 温育 $30 \mathrm{~min}$, 然后如上述 方法测定酶的活性.

\section{2 结果}

\subsection{AdoHcy 水解酶的表达、纯化和活性的测定}

AdoHcy 水解酶按文献 [18]方法在大肠杆菌 JM109 菌株中获得高效表达, 诱导剂 IPTG 的终浓度 为 $0.5 \mathrm{mmol} \cdot \mathrm{L}^{-1}$. 经过 DE-52 离子交换和 Sepharose 4B 凝胶过滤两次色谱柱分离后, 从 $1 \mathrm{~L}$ 细胞培养液 中, 总共得到 AdoHcy 水解酶蛋白约 $45 \mathrm{mg}$. SDSPAGE 电泳分析结果表明产品纯度大于 $85 \%$.

\section{2 不同浓度铜离子对 AdoHcy 水解酶活性的影响}

图 1 表示了 AdoHcy 水解酶与不同浓度铜离子温育 后, 其剩余活性的变化. 可以看到, 随着铜离子浓度的 增加, 酶的活性逐渐降低. 表明铜离子抑制 AdoHcy 水 解酶的活性. 假定铜离子与酶的结合符合 SMichaelis-
Menten 方程, 则铜离子的表观抑制常数 $\left(K_{\mathrm{i}(1)}\right)$ 经用 Microcal Origin ${ }^{\mathrm{TM}}$ 程序计算为 $(14 \pm 4) \mathrm{nmol} \cdot \mathrm{L}^{-1}$.

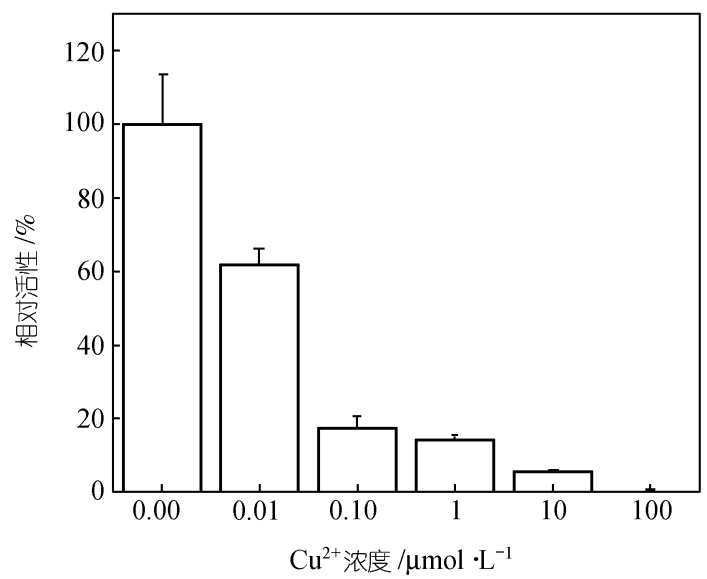

图 1 不同 $\mathrm{Cu}^{2+}$ 浓度温育后 $\mathrm{AdoHcy}$ 水解酶的活性变化

\section{3 铜离子与 AdoHcy 水解酶不同的作用时间对酶 活性的影响}

AdoHcy 水解酶与 $1 \mu \mathrm{mol} \cdot \mathrm{L}^{-1}$ 铜离子温育不同 时间后, 其剩余活性表示在图 2 中. 从图 2 可以看到, 酶的活性随着温育时间的增加而迅速降低. 计算机 数据分析表明, 酶活性降低符合一级动力学过程 $\left(r^{2}=\right.$ 0.9930), 铜离子抑制酶活性的表观速率常数 $k_{\mathrm{app}}=$ $(1.08 \pm 0.15) \mathrm{min}^{-1}$.

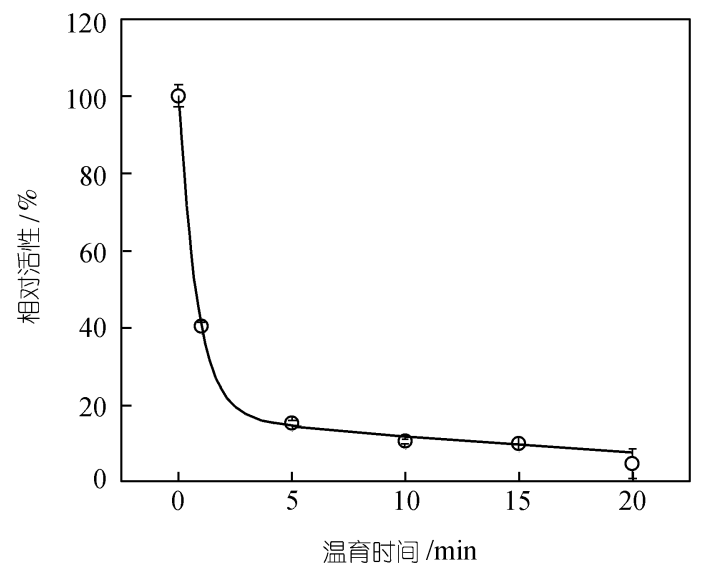

图 $21 \mu \mathrm{mol} \cdot \mathrm{L}^{-1}$ 铜离子不同时间温育后 AdoHcy 水解酶 的活性变化

\section{4 在 Ado 存在下, 不同浓度铜离子对 AdoHcy 水解 酶活性的影响}

图 3 显示了在 $1 \mathrm{mmol} \cdot \mathrm{L}^{-1}$ Ado 存在下, AdoHcy i水解酶与不同浓度铜离子温育后活性的变化以及和 
对照组的比较. 可以看到, 与没有 Ado 存在的结果相 比, $1 \mathrm{mmol} \cdot \mathrm{L}^{-1}$ Ado 存在下酶活性的降低明显要小. 计算机数据分析表明, 在 $1 \mathrm{mmol} \cdot \mathrm{L}^{-1} \mathrm{Ado}$ 的条件下, 铜离子的表观抑制常数 $\left(K_{\mathrm{i}(2)}\right)$ 计算为 $(160 \pm 10)$ $\mathrm{nmol} \cdot \mathrm{L}^{-1}$. 此时, 铜离子抑制酶活性的能力降低至 $1 / 10$ 以下.

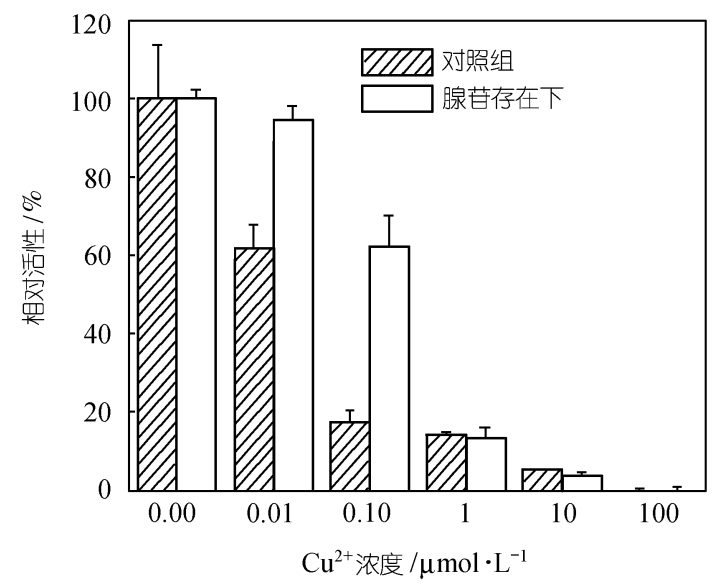

图 $31 \mathrm{mmol} \cdot \mathrm{L}^{-1}$ 腺苷存在下铜离子对 $\mathrm{AdoHcy}$ 水解酶的 活性变化以及与没有腺苷存在下铜离子对 AdoHcy 水解酶 活性影响的比较

空心柱图示 $1 \mathrm{mmol} \cdot \mathrm{L}^{-1}$ Ado 存在下, 条纹柱图示没有 Ado 存在下的 AdoHcy 水解酶的残余活性

\section{3 讨论}

由实验结果(图 1)可以看到, $\mathrm{Cu}^{2+}$ 抑制酶的活性 的能力随着 $\mathrm{Cu}^{2+}$ 浓度增加而增加, 即 $\mathrm{Cu}^{2+}$ 抑制 $\mathrm{AdoHcy}$ 水解酶的活性是一个浓度依赖的过程. 而图 2 结果表 明, $\mathrm{Cu}^{2+}$ 抑制 AdoHcy 水解酶活性是一个较快的但是 时间依赖的过程. 由于 $\mathrm{Cu}(\mathrm{II})$ 一般不生成惰性络合 物, AdoHcy 水解酶分子和 $\mathrm{Cu}^{2+}$ 的结合理论上应该是 瞬间完成的. $\mathrm{Cu}^{2+}$ 抑制 AdoHcy 水解酶活性的时间依 赖性说明 $\mathrm{Cu}^{2+}$ 抑制酶活性不是由于 $\mathrm{Cu}^{2+}$ 的第一配位 结合, 而是其后可能经过酶构象变化等复杂过程, 进 而形成更加特异性的和高稳定性的结合, 从而抑制 酶的活性. 这与文献 [13]报道的有关结果 $\left(\mathrm{Cu}^{2+}\right.$ 与 AdoHcy 水解酶单体 $1: 1$ 结合, 结合常数 $K_{\mathrm{d}}=10^{-16}$ $\left.\mathrm{mol} \cdot \mathrm{L}^{-1}\right)$ 是相一致的.

目前的实验结果还不足以说明 $\mathrm{Cu}^{2+}$ 抑制 AdoHcy 水解酶活性的方式和作用的内在机理. 但图 3 的实验 结果表明其机理显然与影响酶自然底物的结合有关. 图 3 结果显示, 自然底物 Ado 存在时, 可以明显减弱 $\mathrm{Cu}^{2+}$ 抑制酶活性的能力, 其表观解离常数 (即抑制常
数 $K_{\mathrm{i}}$ )大大增加. 由于 $\mathrm{Cu}^{2+}$ 与 $\mathrm{Ado}$ 的络合常数 ${ }^{[21]}$ 仅为 $10^{0.16}, \mathrm{Cu}^{2+}$ 与 $\mathrm{Ado}$ 的配位结合的影响是可以忽略不记 的. 因此, Ado 减弱 $\mathrm{Cu}^{2+}$ 抑制酶活性的能力的原因可 能是底物和铜离子相互竞争与酶结合, 这一有趣现象 在金属离子和酶分子的相互作用中首次被观察到. 由 于铜离子不是自然底物的结构类似物, 因此铜离子和 底物分子在结合过程中的竞争, 与底物的结构类似物 抑制剂是不同的. 从 $K_{\mathrm{i}(2)} / K_{\mathrm{i}(1)}=160 / 14<<1+[\mathrm{Ado}] / K_{\mathrm{d}}$ $=1+1000 / 10$ 的结果验证了两者的不同. 不过, 底物 与 $\mathrm{Cu}^{2+}$ 竞争的现象至少表明 $\mathrm{Cu}^{2+}$ 的结合部位很可能 是在酶的底物结合部位.

生物大分子与 $\mathrm{Cu}^{2+}$ 的稳定结合往往需要蛋白质 组氨酸残基的参与. 观察 AdoHcy 水解酶的晶体结 构 ${ }^{[5,6]}$ 可以发现, 在酶的底物结合位置(图 4), 存在有 His55, His301, Asp131, Asp190 和 Glu156 等可提供配 位原子, 这些残基分散在一个较大的底物可结合的 空腔内. 其中 His55, His301 和 Asp131 靠近, 是最可 能的 $\mathrm{Cu}^{2+}$ 第一结合位点. 因此, 酶的晶体结构表明 $\mathrm{Cu}^{2+}$ 在底物结合部位结合是非常合理的, 但要达到 高度紧密的结合还需要酶构象的一些调整和变化. 这与上述的实验结果相符合.

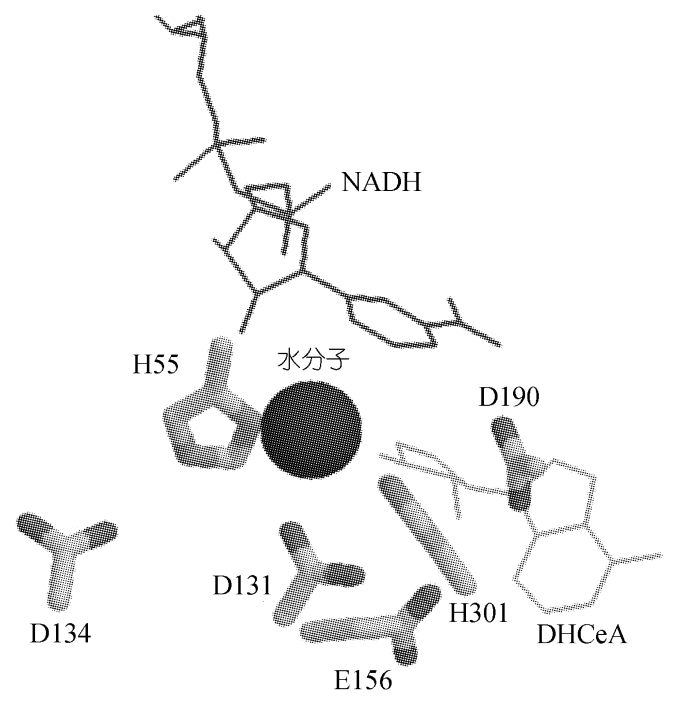

图 4 AdoHcy 水解酶可能结合铜离子的位置示意图 中心小球(水分子)示可能的最初铜离子的位置. 蛋白质晶体结构来源于 文献[5]

\section{4 结论}

$\mathrm{Cu}^{2+}$ 可以抑制 AdoHcy 水解酶活性. 其作用机理 可能是由于 $\mathrm{Cu}^{2+}$ 结合于酶的活性中心并引起酶的构 像变化, c 进而导致酶不能结合底物而失活. 因此, 
$\mathrm{Cu}^{2+}$ 如何与酶分子结合以及如何导致后续变化是 $\mathrm{Cu}^{2+}$ 抑制 AdoHcy 水解酶活性的作用机理的核心问题，尚 需要进一步的研究.

致谢 本工作为中华医学基金专项人才基金、教育部留学 回国人员启动基金和北京大学医学部留学回国人员启动 基金资助项目.

\section{参考文献}

1 Yin D, Yang X, Yuan C, et al. Mechanism-based S-adenosyl-Lhomocystein hydrolase inhibitors in the search for broad-spectrum antiviral agents. Biochemistry: Applying Chemical Principles to the Understanding and Treatment of Diseases. New York: John Wiley \& Sons Inc, 2000. 41 71

2 Turner M, Yang X, Yin D, et al. Structure and function of S-adenosylhomocysteine hydrolase. Cell Biochem Biophy, 2000, 33(2): 101 125

3 Tsunenobu T, Kyu H H, Yasuharu M, et al. Folate and homocysteine metabolism in copper-deficient rats. Biochimica et Biophsica Acta, 1999, 1427: 351 356

4 房静远. DNA 甲基化水平降低与消化系肿瘤. 国外医学 - 消化 系疾病分册, 1994, 14(4): 198 201

5 Turner M, Yuan C, Borchardt R T, et al. Structure determination of selenomethionyl S-adenosylhomocysteine hydrolase using data at a single wavelength. Nat Struct Biol, 1998, 5: 369 376

$6 \mathrm{Hu} \mathrm{Y,} \mathrm{Komoto} \mathrm{J,} \mathrm{Huang} \mathrm{Y,} \mathrm{et} \mathrm{al.} \mathrm{Crystal} \mathrm{structure} \mathrm{of} \mathrm{S-adeno-}$ sylhomocysteine hydrolase from rat liver. Biochemistry, 1999, 38: $8323 \sim 8333$

7 Palmer J L, Abeles R H. The mechanism of action of S-adenosylhomocysteinase. J Biol Chem, 1979, 254: 1217 1226

8 Abeles R H, Tashjian A H, Fish S. The mechanism of inactivation of S-adenosylhomocystein by 2'-deoxyadenosine. Biochem Biophys Res Commun, 1980, 95: 612 617

9 Porter D J, Boyd F L. Mechanism of bovine liver S-adenosylhomocysteine hydrolase. Steady-state and pre-steady-state kinetic analysis. J Biol Chem, 1991, 266: 21616 21625
10 Porter D J, Boyd F L. Reduced S-adenosylhomocysteine hydrolase. Kinetics and thermodynamics for binding of 3 '-ketoadenosine, adenosine, and adenine. J Biol Chem, 1992, 267: 3205 3213

11 Porter D J. S-adenosylhomocysteine hydrolase. Stereochemistry and kinetics of hydrogen transfer. J Biol Chem, 1993, 268: 66 73

12 Suarez J, Chagoya de Sanchez V. Inhibition of S-adenosyl-Lhomocystein hydrolase by adrenaline in isolated guinea-pig papillary muscles. Int J Biochem Cell Biol, 1997, 29: 1279 1284

13 Kloor D, Fuchs S, Petroktistis F, et al. Effects of ions on adenosine binding and enzyme activity of purified S-adenosyl-L-homocystein hydrolase from bovine kidney. Biochemical Pharmacology, 1998, 56: $1493 \sim 1496$

14 Bethin K E, Petrovic N, Ettinger M J. Identification of a major hepatic copper binding protein as S-adenosylhomocysteine hydrolase. J Biol Chem, 1995, 270: 20698 20702

15 Petrovic N, Comi A, Ettinger M J. Copper incorporation into superoxide dismutase in menkes lymphoblasts. J Biol Chem, 1996, 271: $28335 \sim 28340$

16 Bethin K E, Cimato T R, Ettinger M J. Copper binding to mouse liver S-adenosylhomocysteine hydrolase and the effects of copper on its levels. J Biol Chem, 1995, 270: 20703 20711

17 Hultberg B, Andersson A, Isaksson A. Copper ions differ from other thiol reactive metal ions in their effects on the concentration and redox status of thiols in Hela cell cultures. Toxicology, 1997, 117: 89 97

18 Mansoor M A, Bergmark C, Haswell S J, et al. Correlation between plasma total homocysteine and copper in patients with peripheral vascular disease. Clin Chem, 2000, 46: 385 391

19 Yuan C S, Yeh J, Liu S, et al. Mechanism of inactivation of Sadenosylhomocysteine hydrolase by (Z)-4',5'-didehydro-5'-deoxy5'-fluoroadenosine. J Biol Chem , 1993, 268: 17030 17037

20 Robert K. Protein Method. New York: Springer-Verlag, 1982. 239 243

21 Kazakov S A. Nucleic acid binding and catalysis by metal ions. In: Hecht S M, ed. Bioorganic Chemistry: Nucleic Acid. New York: Oxford University Press Inc, 1996. 244 287

(2001-10-08 收稿, 2002-01-31 收修改稿) 